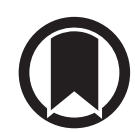

CrossMark

\title{
Peripheral blood leukocyte telomere length is associated with survival of sepsis patients
}

\author{
Shuo Liu (1) ${ }^{1,2,3}$, Chunxue Wang ${ }^{4}$, Gary Green ${ }^{1}$, Hanjing Zhuo ${ }^{1}$, Kathleen D. Liu ${ }^{5}$, \\ Kirsten N. Kangelaris ${ }^{6}$, Antonio Gomez ${ }^{1}$, Alejandra Jauregui ${ }^{1}$, Kathryn Vessel ${ }^{7}$, \\ Serena Ke ${ }^{1}$, Carolyn Hendrickson ${ }^{1}$, Michael A. Matthay ${ }^{1}$, Carolyn S. Calfee ${ }^{1}$, \\ Lorraine B. Ware ${ }^{4}$ and Paul J. Wolters ${ }^{1}$
}

Affiliations: ${ }^{1}$ Division of Pulmonary, Critical Care, Allergy and Sleep Medicine, Dept of Medicine, University of California, San Francisco, San Francisco, CA, USA. ${ }^{2}$ Dept of Respiratory Medicine, The Fourth Affiliated Hospital of China Medical University, Shenyang, Liaoning, People's Republic of China. ${ }^{3}$ Dept of Respiratory Medicine, The First Affiliated Hospital of China Medical University, Shenyang, Liaoning, People's Republic of China. ${ }^{4}$ Dept of Medicine and Pathology, Microbiology and Immunology, Vanderbilt University School of Medicine, Nashville, TN, USA. ${ }^{5}$ Division of Nephrology, Dept of Medicine, University of California, San Francisco, San Francisco, CA, USA. ${ }^{6}$ Division of Hospital Medicine, Dept of Medicine, University of California, San Francisco, San Francisco, CA, USA. ${ }^{7}$ The Cardiovascular Research Institute, University of California, San Francisco, San Francisco, CA, USA.

Correspondence: Paul J. Wolters, University of California, San Francisco, Box 0111, San Francisco, CA 941430111, USA. E-mail: paul.woltersducsf.edu

@ERSpublications

Shorter peripheral blood leukocyte telomere length is strongly associated with worse survival and more severe ARDS in critically ill patients with sepsis http://bit.ly/2mWHKxf

Cite this article as: Liu S, Wang C, Green G, et al. Peripheral blood leukocyte telomere length is associated with survival of sepsis patients. Eur Respir J 2020; 55: 1901044 [https://doi.org/10.1183/13993003.010442019].

ABSTRACT Shorter peripheral blood leukocyte (PBL) telomere length (TL) has been associated with poor outcomes in various chronic lung diseases. Whether PBL-TL is associated with survival from critical illness was tested in this study.

We analysed data from a prospective observational cohort study of 937 critically ill patients at Vanderbilt University Medical Center (VUMC). PBL-TL was measured using quantitative PCR of DNA isolated from PBLs. Findings were validated in an independent cohort of 394 critically ill patients with sepsis admitted to the University of California San Francisco (UCSF).

In the VUMC cohort, shorter PBL-TL was associated with worse 90-day survival (adjusted hazard ratio (aHR) 1.3, 95\% CI 1.1-1.6 per $1 \mathrm{~kb}$ TL decrease; $\mathrm{p}=0.004$ ); in subgroup analyses, shorter PBL-TL was associated with worse 90-day survival for patients with sepsis (aHR 1.5, 95\% CI 1.2-2.0 per $1 \mathrm{~kb}$ TL decrease; $\mathrm{p}=0.001$ ), but not trauma. Although not associated with development of acute respiratory distress syndrome (ARDS), among ARDS subjects, shorter PBL-TL was associated with more severe ARDS (OR 1.7, 95\% CI 1.2-2.5 per $1 \mathrm{~kb}$ TL decrease; $\mathrm{p}=0.006$ ). The associations of PBL-TL with survival (adjusted HR 1.6, 95\% CI 1.2-2.1 per $1 \mathrm{~kb}$ TL decrease; $\mathrm{p}=0.003$ ) and risk for developing severe ARDS (OR 2.5, 95\% CI $1.1-6.3$ per $1 \mathrm{~kb}$ TL decrease; $\mathrm{p}=0.044$ ) were validated in the UCSF cohort.

Short PBL-TL is strongly associated with worse survival and more severe ARDS in critically ill patients, especially patients with sepsis. These findings suggest that telomere dysfunction may contribute to outcomes from critical illness. 


\section{Introduction}

Critical illness is commonly associated with a state of catabolic stress, during which the patient exhibits signs of a systemic inflammatory response. This response is associated with complications, such as the acute respiratory distress syndrome (ARDS), systemic infection, multiorgan failure and death [1,2]. Sepsis and multisystem organ failure remain leading causes of death in critically ill patients, occurring in up to $50 \%$ of patients $[3-5]$.

Telomeres are nucleoprotein structures that protect the ends of chromosomes from degradation $[6,7]$. Telomere length progressively shortens with cell division, ageing and exposure to various genotoxic stresses such as ionising radiation or reactive oxygen species [8]. When critically shortened, telomeres lose their shelterin complex, a protective protein-capping structure [7, 9]. Uncapping exposes a telomere's blunt end, activating a DNA damage response, which leads to either cellular senescence or cell death via apoptosis [10]. Although each cell has 92 telomeres, uncapping a single telomere may be sufficient to activate a DNA damage response [11].

Telomere length may be measured using a variety of methods including Southern blots, flow-FISH (fluorescent in situ hybridisation) or quantitative (q)PCR [12]. Telomere lengths reported by these methods represent the average telomere length within the population of analysed cells. The shorter the average telomere length, the greater the probability that cells within the population have critically short telomeres. Furthermore, average telomere length within a tissue can be a surrogate marker of the probability of short telomeres in other tissues within the same individual [13]. Considering these relationships, peripheral blood leukocyte (PBL) telomere lengths (TLs) have been analysed in large epidemiological studies in which shorter telomere lengths have been shown to be associated with mental illness, atherosclerotic cardiovascular disease and pulmonary fibrosis [12].

Short PBL-TL is associated with worse survival in patients with idiopathic pulmonary fibrosis (IPF) [14], where telomere dysfunction is increasingly recognised as a molecular driver of the disease [15]. Patients with IPF can suffer from acute exacerbations of their underlying disease. These exacerbations are characterised pathologically by diffuse alveolar damage [16], which is also the pathologic finding in ARDS $[17,18]$. In addition, telomere dysfunction isolated to alveolar epithelial cells increases susceptibility of mice to influenza infection, and telomere dysfunction isolated to mesenchymal cells causes pulmonary oedema in mice [19]. Based on the murine studies of lung telomere dysfunction and the histopathological relationship between acute exacerbations of IPF and ARDS, we pursued the hypothesis that PBL-TL may also be associated with survival of critically ill patients, including those with ARDS.

\section{Material and methods}

Study design and patient populations

We analysed data from two prospective observational cohort studies of critically ill patients, one from the Vanderbilt University Medical Center (VUMC, Nashville, TN, USA) and one from the University of California San Francisco (UCSF, San Francisco, CA, USA).

The VUMC cohort comprised patients aged $\geqslant 18$ years admitted to the medical, surgical, trauma or cardiovascular intensive care units (ICUs) from January 2006 to September 2013. All patients who were not discharged from the ICU by ICU day 2 were eligible for enrolment on the morning of ICU day 2 , unless they had chronic lung disease, uncomplicated overdose, imminent death or had undergone cardiothoracic surgery. DNA was isolated from PBLs collected the morning after ICU admission. In the UCSF cohort, patients with sepsis admitted to the ICU from the emergency department from September 2009 to January 2017 were eligible, excluding patients with trauma or isolated neurological conditions, with the whole-blood samples collected within $24 \mathrm{~h}$ of admission to the ICU.

In both cohorts, the diagnosis of ARDS was made using clinical data including two-physician consensus review of chest radiographs for bilateral opacities, in accordance with the Berlin definition of ARDS [17]. The severity of ARDS was classified based on degree of hypoxaemia: mild (arterial oxygen tension $\left(P_{\mathrm{aO}_{2}}\right) /$ inspiratory oxygen fraction $\left(F_{\mathrm{IO}_{2}}\right)$ ratio $\left.>200-300 \mathrm{mmHg}\right)$, moderate $\left(P_{\mathrm{aO}_{2}} / F_{\mathrm{IO}_{2}}>100-200 \mathrm{mmHg}\right)$ and severe $\left(P_{\mathrm{aO}_{2}} / F_{\mathrm{IO}_{2}} \leqslant 100 \mathrm{mmHg}\right)$ [17]. Patients were followed for ARDS for the first four ICU days at Vanderbilt and the first five ICU days at UCSF. For all participants, the presence of sepsis was determined prospectively by a trained study clinician based on the presence of suspected or documented infection plus two or more systemic inflammatory response syndrome criteria. Demographic and clinical information, including APACHE (Acute Physiology and Chronic Health Evaluation) II score and ARDS risk factors were collected. Outcome data included 90-day mortality and 1-year mortality (60-day in-hospital mortality for UCSF sepsis cohort), need for dialysis, duration of mechanical ventilation, duration of ICU stay and duration of hospital stay. Patients with DNA of insufficient quality were excluded from the study. 
Ethics approval was obtained from institutional committees at VUMC and at UCSF for use of the source databases and biospecimens. Written informed consent was obtained for all patients with the following exceptions. At VUMC, the institutional review board granted a waiver of consent in cases where the patient was unable to consent and no surrogate could be identified. At UCSF, the institutional review board granted a waiver of consent for patients who died before consent could be obtained [20].

\section{PBL-TL measurements}

In both cohorts, PBL genomic DNA was isolated for patients using Gentra Puregene cell kit (Qiagen, Valencia, CA, USA). DNA was visualised on agarose gels to determine quality, and degraded DNA samples were excluded from analysis. Average individual PBL-TLs were measured in triplicate by personnel blinded to all clinical data using quantitative uniplex qPCR and the acidic ribosomal phosphoprotein 36B4 gene as a reference housekeeping gene, as described previously [14, 21-23]. Average telomere lengths were determined by subtracting telomere and reference median cycle threshold (CT) values. Telomere lengths were calculated by comparison to reference samples [24]. Using these methods, the intra-assay coefficient of variation $(\mathrm{CV})$ was $<1 \%$, the interassay $\mathrm{CV}$ was $<3 \%$ and the intraclass correlation coefficient for triplicate measurements was 0.981 (95\% CI 0.973-0.986).

\section{Statistical analysis}

Clinical characteristics of the study cohorts were compared using Kruskal-Wallis rank sum test for continuous variables and Fisher's exact tests for categorical variables. Logistic regression models were utilised to examine the relationship between PBL-TL and hospital mortality controlling for prespecified cofounders reported to influence telomere lengths including age, sex, ethnicity, smoking history and APACHE II. We used ordinal regression models to evaluate the association between PBL-TL and severity of ARDS. Kaplan-Meier plots were constructed to compare patient survival by quartile of PBL-TL, and survival rates in different groups were compared by a log-rank test. The association between PBL-TL and survival time was estimated using Cox proportional hazards regression adjusting for age, sex, ethnicity, smoking history and APACHE II. The proportionality of hazards assumption was checked by Schoenfeld residuals plots against time for each covariate. Cubic splines were applied to permit nonlinear associations and the best regression models were selected based upon the Akaike information criterion to avoid overfitting. Missing values were imputed using transcan function in the $\mathrm{R}$ Hmisc package. Variance inflation factor was used to check multicollinearity among variables in the models. All analyses were performed using the R software version 3 (www.r-project.org).

\section{Results}

\section{Discovery cohort characteristics}

The VUMC cohort consisted of 937 critically ill patients. The mean \pm sD age was $53 \pm 17$ years, and $60 \%$ were male (table 1). $72 \%$ of patients were enrolled within $24 \mathrm{~h}$ of hospital admission. Comparison of clinical characteristics between ARDS patients and non-ARDS patients is shown in supplementary table S1. In subgroup analysis, several subcohorts were analysed including a trauma cohort consisting of 281 patients, an ARDS cohort consisting of 249 patients, a sepsis cohort consisting of 368 patients, and a sepsis with ARDS cohort consisting of 151 patients. Some patients were included in more than one subgroup (supplementary figure $\mathrm{S} 1$ ).

\section{Relationship between PBL-TL and survival of critically ill patients}

In the VUMC cohort, the median PBL-TL was $6.96 \mathrm{~kb}$ pairs (interquartile range (IQR) $6.47-7.41 \mathrm{~kb}$ pairs) (supplementary figure S2); shorter PBL-TLs were independently associated with worse 90-day survival and 1-year survival for all critically ill patients (for 90-day survival: unadjusted hazard ratio (HR) 1.6, 95\% CI 1.3-1.8 per $1 \mathrm{~kb}$ TL decrease; $\mathrm{p}<0.0001$; adjusted HR 1.3 , 95\% CI $1.1-1.5$ per $1 \mathrm{~kb}$ TL decrease; $\mathrm{p}=0.004$; for 1-year survival: unadjusted HR 1.5, 95\% CI 1.3-1.8 per $1 \mathrm{~kb}$ TL decrease; $\mathrm{p}<0.0001$; adjusted HR 1.3, 95\% CI 1.1-1.5 per $1 \mathrm{~kb}$ TL decrease; $\mathrm{p}=0.004$ ) (table 2). Among patients with PBL-TL below the 25th percentile, $34 \%$ died within 90 days of their critical illness compared to $15 \%$ of patients with PBL-TL above the 75th percentile (figure 1). Shorter PBL-TL was significantly associated with increased 90-day mortality, and 1-year mortality in adjusted models both in the overall VUMC cohort and sepsis subgroup (supplementary table S2). The 90 -day mortality rate increased $36 \%$ for every $1 \mathrm{~kb}$ decrease in PBL-TL in VUMC overall cohort, after adjusting for other covariates (supplementary table S2 and figure 2).

\section{Relationship between PBL-TL and survival of trauma patients}

In the VUMC cohort, critically ill trauma patients had significantly longer PBL-TLs compared to non-trauma patients after adjustment for age $(\mathrm{p}<0.0001)$. There was no relationship between PBL-TL and the survival of trauma patients (adjusted HR 0.9, 95\% CI 0.8-1.7 per $1 \mathrm{~kb}$ TL decrease; $\mathrm{p}=0.59$ ) (supplementary table S3). 


$\begin{array}{lc}\text { TABLE } 1 \text { Clinical characteristics of critically ill patients in the Vanderbilt University Medical } \\ \text { Center cohort } & 937 \\ \text { Subjects } & 53 \pm 17 \\ \text { Age years } & 563(60) \\ \text { Male } & 172(18) \\ \text { Non-Caucasian } & 29 \pm 8.3 \\ \text { BMI kg-m }{ }^{-2} & \\ \text { Smoking status } & 355(38) \\ \text { None } & 212(23) \\ \text { Former smoker } & 307(33) \\ \text { Current smoker } & 63(6.7) \\ \text { Unknown } & \\ \text { ARDS risk factor } & 216(23) \\ \text { Sepsis } & 105(11) \\ \text { Pneumonia } & 275(30) \\ \text { Severe trauma } & 57(6.2) \\ \text { Aspiration } & 272(29) \\ \text { Others } & 219 \pm 123 \\ \boldsymbol{P}_{\text {ao }_{2} / F_{\mathbf{I}_{2}} \text { ratio }} & 25.7 \pm 8.06 \\ \text { APACHE II score } & \\ \text { Organ failure } & 591(63.1) \\ \text { Circulatory failure } & 186(19.9) \\ \text { Coagulation failure } & 133(14.2) \\ \text { Hepatic failure } & 246(26) \\ \text { Renal failure } & 358(38.2) \\ \text { Vasopressors } & 6.95 \pm 0.79 \\ \text { Telomere length kb } & \end{array}$

Data are presented as $\mathrm{n}$, mean \pm SD or $\mathrm{n}(\%)$. BMI: body mass index; ARDS: acute respiratory distress syndrome; $P_{\mathrm{aO}_{2}}$ : arterial oxygen tension; $F_{\mathrm{IO}_{2}}$ : inspiratory oxygen fraction; APACHE: Acute Physiology and Chronic Health Evaluation.

TABLE 2 Relationship of peripheral blood leukocytes telomere length to survival in critically ill patients across both Vanderbilt University Medical Center (VUMC) cohort and University of California San Francisco (UCSF) sepsis cohort using Cox proportional hazards models

\begin{tabular}{|c|c|c|c|}
\hline & \multicolumn{3}{|c|}{ Telomere length per $1 \mathrm{~kb}$ decrease } \\
\hline & Subjects $n$ & HR $(95 \% \mathrm{CI})$ & p-value \\
\hline VUMC critically ill cohort & 937 & & \\
\hline \multicolumn{4}{|l|}{ 90-day survival } \\
\hline Unadjusted & & $1.56(1.32-1.83)$ & $<0.0001$ \\
\hline Adjusted ${ }^{\#}$ & & $1.30(1.08-1.56)$ & 0.004 \\
\hline \multicolumn{4}{|l|}{ 1-year survival } \\
\hline Unadjusted & & $1.53(1.33-1.78)$ & $<0.0001$ \\
\hline Adjusted ${ }^{\#}$ & & $1.27(1.08-1.49)$ & 0.004 \\
\hline VUMC sepsis subgroup & 368 & & \\
\hline \multicolumn{4}{|l|}{ 90-day survival } \\
\hline Unadjusted & & $1.61(1.27-2.06)$ & $<0.0001$ \\
\hline Adjusted ${ }^{\#}$ & & $1.54(1.19-2.00)$ & 0.001 \\
\hline \multicolumn{4}{|l|}{ 1-year survival } \\
\hline Unadjusted & & $1.51(1.22-1.86)$ & 0.0001 \\
\hline Adjusted $^{\#}$ & & $1.41(1.12-1.77)$ & 0.003 \\
\hline UCSF sepsis cohort & 394 & & \\
\hline \multicolumn{4}{|l|}{ 60-day survival } \\
\hline Unadjusted & & $1.53(1.19-1.98)$ & 0.001 \\
\hline Adjusted $^{\#}$ & & $1.57(1.17-2.12)$ & 0.003 \\
\hline
\end{tabular}



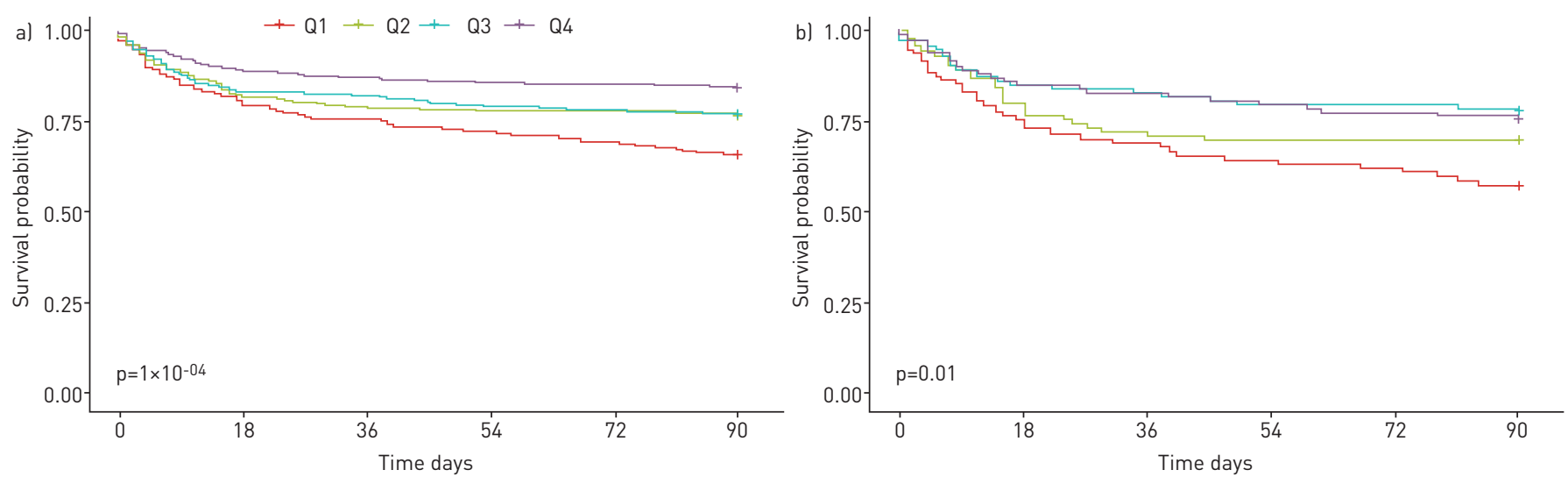

Number at risk (number of events)

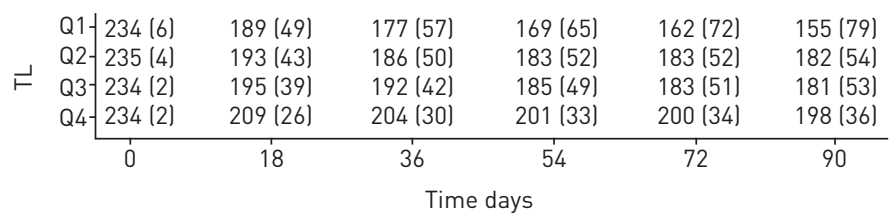

Number at risk (number of events)

\begin{tabular}{cccccc} 
Q1- $92(3)$ & $69(25)$ & $63(29)$ & $59(33)$ & $57(35)$ & $53(39)$ \\
$\qquad 2-92(0)$ & $73(22)$ & $66(27)$ & $64(28)$ & $64(28)$ & $64(28)$ \\
Q3- $92(3)$ & $78(14)$ & $76(16)$ & $73(19)$ & $73(19)$ & $72(20)$ \\
Q4- $92(1)$ & $78(14)$ & $76(16)$ & $73(19)$ & $71(21)$ & $70(22)$ \\
\hline 0 & 18 & 36 & 54 & 72 & 90 \\
\hline \multicolumn{5}{c}{ Time days }
\end{tabular}

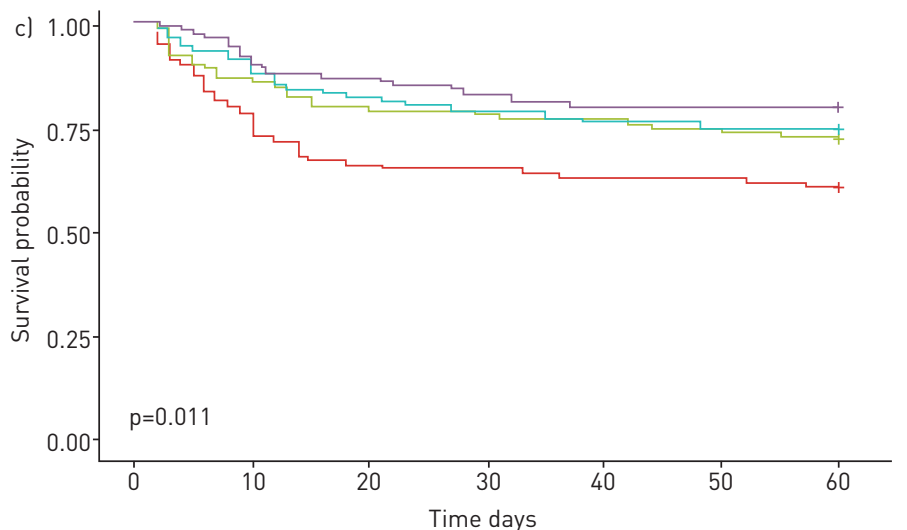

Number at risk (number of events)

\begin{tabular}{|c|c|c|c|c|c|c|c|}
\hline \multirow[t]{5}{*}{ Q1 } & $99(0)$ & 77 (27) & 65 (34) & $64(35)$ & 62 (37) & 62 (37) & 60 (39) \\
\hline & $98(0)$ & 85 (14) & $78(21)$ & $76(22)$ & 75 (23) & 73 (26) & 71 (27) \\
\hline & $99(0)$ & 90 (12) & 81 (18) & $78(21)$ & 75 (24) & 74 (25) & 74 (25) \\
\hline & $98(0)$ & $90(10)$ & 85 (13) & 81 (17) & $78(20)$ & 78 (20) & $78(20)$ \\
\hline & 0 & 10 & 20 & 30 & 40 & 50 & 60 \\
\hline
\end{tabular}

FIGURE 1 Shorter telomere lengths are associated with worse survival for critically ill patients in the Vanderbilt University Medical Center (VUMC) cohort, VUMC sepsis subgroup and University of California San Francisco (UCSF) sepsis cohort. a) VUMC cohort (n=937); b) VUMC sepsis subgroup ( $\mathrm{n}=368)$; c) UCSF sepsis cohort $(\mathrm{n}=394)$. Estimated survival for critically ill patients stratified by telomere length (TL) quartiles for survival. Q4: longest quartile; Q3: 50-75\%; Q2: 25-50\%; Q1: shortest quartile.

Relationship between PBL-TL and the development of ARDS or organ failure

In the overall VUMC cohort, PBL-TL was not significantly different between ARDS and non-ARDS patients (supplementary table S1), and PBL-TL was not associated with the development of ARDS $(\mathrm{p}=0.87)$. In multivariate ordinal logistic regression models, among ARDS patients in the overall VUMC cohort, shorter PBL-TLs were associated with increased risk for developing severe ARDS (table 3). In exploratory univariate analysis, PBL-TL was associated with the number of failed organs $(\mathrm{p}=0.02)$ and vasopressor use $(\mathrm{p}=0.01)$, but not the need for mechanical ventilation $(\mathrm{p}=0.78)$ (supplementary figure $\mathrm{S} 3$ ). These associations did not persist after adjusting for covariates.

Relationship between PBL-TL and outcomes of sepsis patients

In the VUMC cohort, after adjustment for relevant individual covariates (age, male sex, ethnicity, smoking status and APACHE II score), sepsis patients had significantly shorter PBL-TLs than nonsepsis patients $(\mathrm{p}=0.0007)$; shorter PBL-TL was independently associated with worse 90-day survival and 1-year survival 

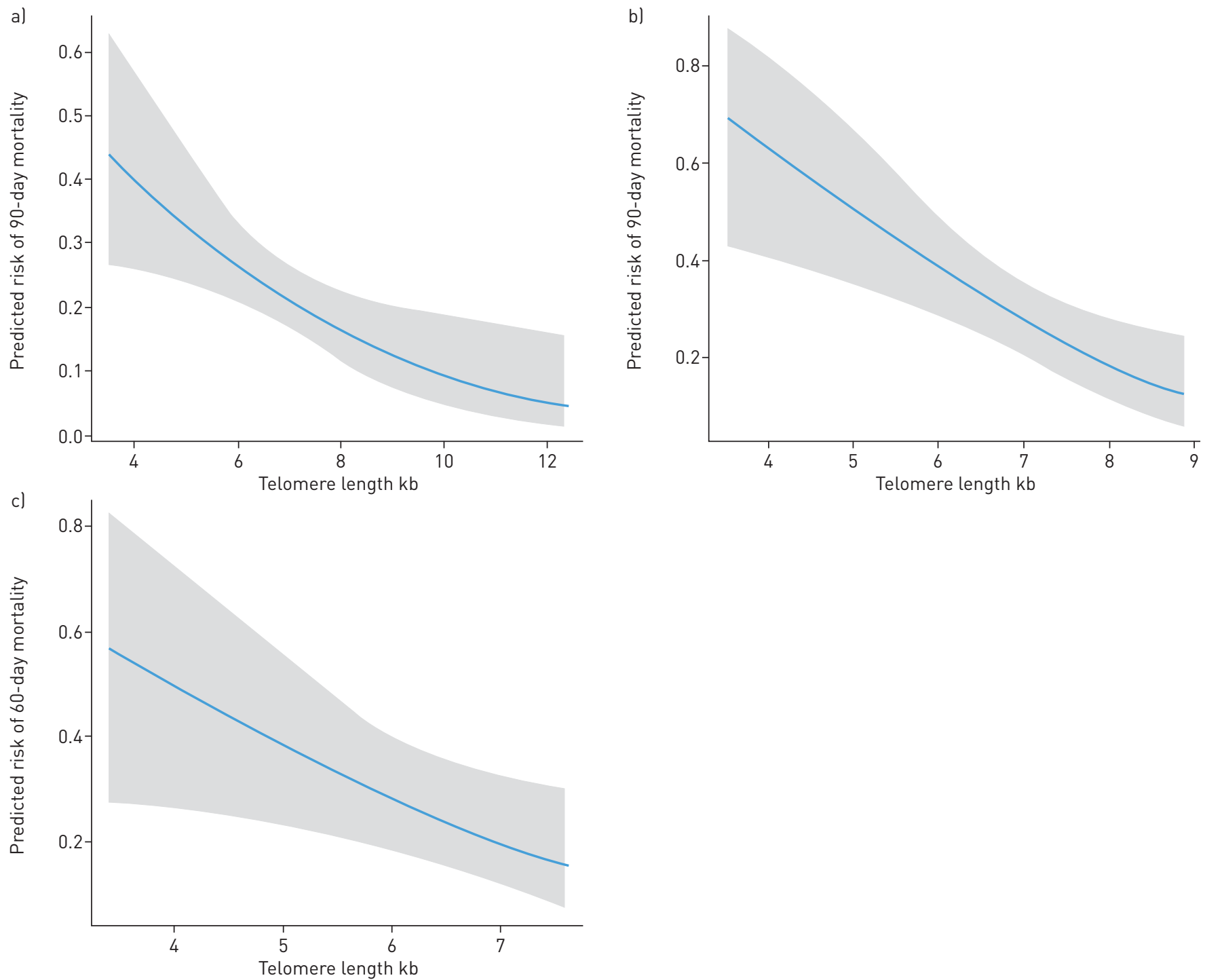

FIGURE 2 Relationship between peripheral blood leukocyte telomere lengths and mortality for critically ill patients in a logistic regression model. a) Vanderbilt University Medical Center (VUMC) cohort; b) VUMC sepsis subgroup; c) University of California San Francisco sepsis cohort. Model controlled for age, male sex, ethnicity, smoking status and Acute Physiology and Chronic Health Evaluation (APACHE) II score.

of sepsis patients (for 90-day survival: unadjusted HR 1.6, 95\% CI 1.3-2.1 per $1 \mathrm{~kb}$ TL decrease; $\mathrm{p}<0.0001$; adjusted HR 1.5, 95\% CI 1.2-2.0 per $1 \mathrm{~kb}$ TL decrease; $\mathrm{p}=0.001$; for 1-year survival: unadjusted HR 1.5, 95\% CI 1.2-1.9 per $1 \mathrm{~kb}$ TL decrease; $\mathrm{p}=0.0001$; adjusted HR 1.4, 95\% CI $1.1-1.8$ per $1 \mathrm{~kb}$ TL decrease; $\mathrm{p}=0.003$ ) (table 2 and figure 2). In the VUMC sepsis subgroup, $42 \%$ of patients with PBL-TL below the 25th percentile died within 90 days of enrolment compared to $24 \%$ of patients with PBL-TL above the 75th percentile (figure 1). Using multivariate ordinal logistic regression models, among ARDS patients in the VUMC sepsis cohort, shorter PBL-TLs were associated with an increased risk of developing severe ARDS (table 3). There was no significant association between PBL-TL and the number of failed organs, vasopressor use or need for mechanical ventilation in the sepsis subgroup (supplementary figure S4).

\section{Validation cohort}

Because PBL-TL was most prominently associated with survival in the VUMC sepsis subgroup, and was not associated with outcomes in the trauma cohort, we next sought to validate these findings in a separate sepsis cohort from UCSF. The UCSF sepsis validation cohort consisted of 394 critically ill patients with sepsis; comparison of the UCSF sepsis patients to the VUMC sepsis patients is shown in supplementary table S4. In the UCSF sepsis validation cohort, the median PBL-TL was 6.25 (IQR 5.87-6.58) kb pairs (supplementary figure S2). Shorter PBL-TL was independently associated with worse 60-day survival 
TABLE 3 Association of acute respiratory distress syndrome (ARDS) severity with peripheral blood leukocyte telomere length in multivariate ordinal logistic regression models

\begin{tabular}{lccccc} 
Multivariable analysis (individual covariates) & \multicolumn{2}{c}{ VUMC cohort } & & & VUMC sepsis subgroup \\
\cline { 2 - 3 } \cline { 6 - 7 } & OR (95\% Cl) & p-value & & OR (95\% CI) & p-value \\
\hline Subjects $\mathbf{n}$ & 249 & & & 135 & \\
Age & $0.97(0.95-0.99)$ & 0.0005 & & $0.97(0.94-0.99)$ & 0.005 \\
Male sex & $0.68(0.41-1.13)$ & 0.14 & & $0.65(0.31-1.34)$ & 0.24 \\
Ethnicity (non-Caucasian) & $1.02(0.50-2.11)$ & 0.95 & & $0.74(0.25-2.20)$ & 0.58 \\
Smoking status (never-smoking) & $2.42(1.43-4.10)$ & 0.001 & & $2.58(1.22-5.45)$ & 0.01 \\
APACHE II score & $1.10(1.06-1.15)$ & $<0.0001$ & & $1.06(1.01-1.12)$ & 0.0004 \\
Telomere length per 1 kb decrease & $1.71(1.16-2.50)$ & 0.006 & & $2.52(1.40-4.56)$ & 0.002
\end{tabular}

The dependent variable is severity of ARDS with three levels: mild, moderate or severe. VUMC: Vanderbilt University Medical Center; APACHE: Acute Physiology and Chronic Health Evaluation.

(unadjusted HR 1.5, 95\% CI 1.2-2.0 per $1 \mathrm{~kb}$ TL decrease; $\mathrm{p}=0.001$; adjusted HR 1.6, 95\% CI $1.2-2.1$ per $1 \mathrm{~kb}$ TL decrease; $\mathrm{p}=0.003$ ) (table 2 and figure 2). 39\% of patients with PBL-TL below the 25th percentile died within 60 days of their critical illness compared to $20 \%$ of patients with PBL-TL above the 75 th percentile (figure 1). In addition, shorter PBL-TLs were associated with an increased risk of developing severe ARDS in the UCSF validation cohort (adjusted OR 2.5, 95\% CI 1.1-6.3 per $1 \mathrm{~kb}$ TL decrease; $\mathrm{p}=0.044)$.

\section{Discussion}

This study reports that shorter PBL-TL is associated with higher mortality among critically ill patients and that this association is strongest among patients with sepsis. Additionally, among patients with ARDS, shorter PBL-TL was associated with more severe lung injury. These findings suggest that telomere dysfunction may contribute to outcomes from critical illness.

Older adults (age $>65$ years) are at increased risk of death due to critical illness including sepsis $[25,26]$. Molecular explanations for this increased risk remain poorly defined. This study is novel, because it examined the relationship between PBL-TL and outcomes in critically ill patients. PBL-TL is a marker of the molecular age of the patient, with the length being influenced by an individual's chronological age [8], genetic background [27] and lifetime exposure to environmental insults that contribute to telomere attrition. Therefore, these findings suggest that telomere dysfunction in individuals with an advanced molecular age may contribute to critical illness outcomes. The relevant cell types and molecular mediators of this telomere dysfunction that contribute to the poor outcomes will require future study.

The association between short telomeres and increased mortality of critically ill patients was strongest in patients with sepsis. One explanation for the association is that it may be due in part to an impaired capacity of patients with short telomeres to control infections. This hypothesis would be consistent with prior reports that shorter PBL-TL is associated with a higher risk of acquiring infections such as pneumonia [28]. This increased risk of infection may be due to immune cell senescence in leukocytes [29] or T-cell defects such as depleted naive T-cells, increased apoptosis and restricted T-cell repertoire, which have been reported in patients with short telomeres [30]. Alternatively, oxidative stress generated during sepsis could lead to telomere shortening in a progressive positive feedback loop in sepsis, culminating in immune defects and multiple organ failure, which may contribute to poor sepsis survival [31]. Another potential explanation is that the findings are due to differences in the proportion of leukocyte subsets in septic patients. However, when neutrophil count was included as a covariate in the Cox model for survival in a subset of patients from the VUMC cohort with available neutrophil count data, neutrophil count was not found to be a confounder (the p-value for neutrophil count is 0.93).

In the subset of critically ill patients with ARDS, PBL-TL was not associated with the onset of ARDS, but was significantly associated with ARDS severity, with severe ARDS patients having shorter PBL-TLs compared to mild ARDS patients. PBL-TL may be a surrogate marker of an individual patient's risk for short telomeres within cells residing in lung tissue [13]. Telomere dysfunction in alveolar type II cells of mice has been shown to predispose the animals to death from viral pneumonia and mesenchymal cell telomere dysfunction in the lung causes pulmonary oedema [19]. Similarly, patients with IPF, a disease associated with short telomeres in alveolar type II cells, develop acute exacerbations, which are characterised pathologically by diffuse alveolar damage [16]. Putman et al. [32] found that critically ill patients with interstitial lung abnormalities were significantly more likely to develop ARDS. Although the 
pathogenesis of acute lung injury remains incompletely understood, these findings suggest that telomere dysfunction in alveolar type II cells could increase susceptibility of those cells to external stressors and therefore to the development of lung oedema.

There are limitations to this study. Average PBL-TL was measured on genomic DNA samples isolated from blood leukocytes using quantitative PCR. This method has been reported to have a higher interassay coefficient of variation than other methods of telomere length measurements [33]. Nevertheless, the test characteristics of telomere length measurements in our lab are consistent with test characteristics of other methods for measuring telomeres, such as flow-FISH [34]. Additional factors that may influence PBL-TL measurements include DNA integrity or the population of leukocytes from which DNA is extracted [35]. There was a difference in the average PBL-TL between the VUMC and UCSF sepsis cohorts, probably due to the 11-year mean age difference between the two cohorts. We did not find a significant difference in leukocyte subpopulations between groups of critically ill patients of these two cohorts, suggesting that differences in leukocyte populations do not explain the findings. Furthermore, these differences in age range would decrease the probability that the findings would be replicated, and consequently should increase the generalisability of the findings. The majority of patients were enrolled prior to the current Sepsis-3 guidelines [36]. However, the majority of patients had severe sepsis, which is consistent with Sepsis-3 guidelines. Finally, we were unable to test the correlation between PBL-TL and telomere lengths of cellular subtypes in other organs. It may be useful in future studies to examine patient survival in relationship to telomere lengths within cellular subtypes of other organs.

In conclusion, shorter PBL-TL is associated with mortality and ARDS severity in two independent cohorts of critically ill patients, suggesting that telomere dysfunction is associated with the pathobiology of critical illness. Measures of PBL-TL may provide a unique opportunity to advance understanding of the interaction between ageing and the pathobiology of ARDS and sepsis.

Acknowledgements: We are grateful to all the patients and coordinators who participated in this study at all sites.

Author contributions: P.J. Wolters, C.S. Calfee, L.B. Ware and M.A. Matthay designed the study. S. Liu and G. Green performed telomere length measurements. C. Wang and S. Liu analysed the data. H. Zhuo, L.B. Ware, C.S. Calfee, M.A. Matthay, K.D. Liu, K.N. Kangelaris, A. Gomez, A. Jauregui, K. Vessel, S. Ke and C. Hendrickson collected data for the study. S. Liu drafted the manuscript. All authors contributed to study conduct, data interpretation and manuscript preparation.

Conflict of interest: S. Liu reports grants from National Natural Science Foundation of China, during the conduct of the study. C. Wang has nothing to disclose. G. Green has nothing to disclose. H. Zhuo has nothing to disclose. K.D. Liu has nothing to disclose. K.N. Kangelaris has nothing to disclose. A. Gomez has nothing to disclose. A. Jauregui has nothing to disclose. K. Vessel has nothing to disclose. S. Ke has nothing to disclose. C. Hendrickson has nothing to disclose. M.A. Matthay reports grants from NIH/NHLBI (for research and clinical trials of ARDS and sepsis), Department of Defense (to carry out a clinical trial of ARDS) and Bayer Pharmaceuticals (for an observational research study of ARDS), and personal fees from Cerus Therapeutics (ARDS consultation) and CSL Behring (ARDS consultation), outside the submitted work. C.S. Calfee reports grants from NIH, during the conduct of the study; grants from GlaxoSmithKline (for an observational study of sepsis and ARDS), grants and personal fees from Bayer (for an observational study of ARDS and consultancy about ARDS), and personal fees from Prometic (consultancy), CSL Behring (medical advisory board), Roche/Genentech (consultancy) and Quark (consultancy), outside the submitted work. L.B. Ware reports grants from National Institutes of Health, during the conduct of the study; personal fees from Quark Pharmaceuticals (advisory board), CSL Behring (advisory board) and Bayer (advisory board), outside the submitted work. P.J. Wolters reports grants from MedImmune, Genentech and Boehringer Ingelheim, and personal fees from Roche, Boehringer Ingelheim, Blade Therapeutics and Pliant, outside the submitted work.

Support statement: The study was funded in part by NIH grants HL103836 and HL135849 (L.B. Ware), HL131621 and HL140026 (C.S. Calfee), HL51856 (M.A. Matthay), National Natural Science Foundation of China 81700063 (S. Liu), and the Nina Ireland Program for Lung Health. Funding information for this article has been deposited with the Crossref Funder Registry.

\section{References}

1 McClave SA, Taylor BE, Martindale RG, et al. Guidelines for the provision and assessment of nutrition support therapy in the adult critically ill patient: Society of Critical Care Medicine (SCCM) and American Society for Parenteral and Enteral Nutrition (A.S.P.E.N.). JPEN J Parenter Enteral Nutr 2016; 40: 159-211.

2 Fan E, Zakhary B, Amaral A, et al. Liberation from mechanical ventilation in critically ill adults. An official ATS/ ACCP clinical practice guideline. Ann Am Thorac Soc 2017; 14: 441-443.

3 Angus DC, van der Poll T. Severe sepsis and septic shock. N Engl J Med 2013; 369: 840-851.

4 Hotchkiss RS, Monneret G, Payen D. Immunosuppression in sepsis: a novel understanding of the disorder and a new therapeutic approach. Lancet Infect Dis 2013; 13: 260-268.

$5 \quad$ Ware LB, Matthay MA. The acute respiratory distress syndrome. N Engl J Med 2000; 342: 1334-1349.

6 Greider CW, Blackburn EH. Identification of a specific telomere terminal transferase activity in Tetrahymena extracts. Cell 1985; 43: 405-413.

$7 \quad$ Calado RT, Young NS. Telomere diseases. N Engl J Med 2009; 361: 2353-2365. 
8 Suram A, Herbig U. The replicometer is broken: telomeres activate cellular senescence in response to genotoxic stresses. Aging Cell 2014; 13: 780-786.

9 Blackburn EH. Telomere states and cell fates. Nature 2000; 408: 53-56.

10 Liu CC, Ma DL, Yan TD, et al. Distinct responses of stem cells to telomere uncapping - a potential strategy to improve the safety of cell therapy. Stem Cells 2016; 34: 2471-2484.

11 Hemann MT, Strong MA, Hao LY, et al. The shortest telomere, not average telomere length, is critical for cell viability and chromosome stability. Cell 2001; 107: 67-77.

12 Savage SA. Beginning at the ends: telomeres and human disease. F1000Res 2018; 7: F1000 Faculty Rev 524.

13 Daniali L, Benetos A, Susser E, et al. Telomeres shorten at equivalent rates in somatic tissues of adults. Nat Commun 2013; 4: 1597.

14 Stuart BD, Lee JS, Kozlitina J, et al. Effect of telomere length on survival in patients with idiopathic pulmonary fibrosis: an observational cohort study with independent validation. Lancet Respir Med 2014; 2: 557-565.

15 Wolters PJ, Blackwell TS, Eickelberg O, et al. Time for a change: is idiopathic pulmonary fibrosis still idiopathic and only fibrotic? Lancet Respir Med 2018; 6: 154-160.

16 Collard HR, Moore BB, Flaherty KR, et al. Acute exacerbations of idiopathic pulmonary fibrosis. Am J Respir Crit Care Med 2007; 176: 636-643.

17 Ranieri VM, Rubenfeld GD, Thompson BT, et al. Acute respiratory distress syndrome: the Berlin definition. JAMA 2012; 307: 2526-2533.

18 Guerin C, Bayle F, Leray V, et al. Open lung biopsy in nonresolving ARDS frequently identifies diffuse alveolar damage regardless of the severity stage and may have implications for patient management. Intensive Care Med 2015; 41: 222-230.

19 Naikawadi RP, Disayabutr S, Mallavia B, et al. Telomere dysfunction in alveolar epithelial cells causes lung remodeling and fibrosis. JCI Insight 2016; 1: e86704.

20 Agrawal A, Matthay MA, Kangelaris KN, et al. Plasma angiopoietin-2 predicts the onset of acute lung injury in critically ill patients. Am J Respir Crit Care Med 2013; 187: 736-742.

21 Cawthon RM. Telomere measurement by quantitative PCR. Nucleic Acids Res 2002; 30 : e47.

22 Faust HE, Golden JA, Rajalingam R, et al. Short lung transplant donor telomere length is associated with decreased CLAD-free survival. Thorax 2017; 72: 1052-1054.

23 Ley B, Newton CA, Arnould I, et al. The MUC5B promoter polymorphism and telomere length in patients with chronic hypersensitivity pneumonitis: an observational cohort-control study. Lancet Respir Med 2017; 5: 639-647.

24 Listerman I, Sun J, Gazzaniga FS, et al. The major reverse transcriptase-incompetent splice variant of the human telomerase protein inhibits telomerase activity but protects from apoptosis. Cancer Res 2013; 73: 2817-2828.

25 Ferrante LE, Pisani MA, Murphy TE, et al. Functional trajectories among older persons before and after critical illness. JAMA Intern Med 2015; 175: 523-529.

26 Fuchs L, Chronaki CE, Park S, et al. ICU admission characteristics and mortality rates among elderly and very elderly patients. Intensive Care Med 2012; 38: 1654-1661.

27 Garcia CK, Wright WE, Shay JW. Human diseases of telomerase dysfunction: insights into tissue aging. Nucleic Acids Res 2007; 35: 7406-7416.

28 Helby J, Nordestgaard BG, Benfield T, et al. Shorter leukocyte telomere length is associated with higher risk of infections: a prospective study of 75,309 individuals from the general population. Haematologica 2017; 102: 1457-1465.

29 Fritsch RD, Shen X, Illei GG, et al. Abnormal differentiation of memory T cells in systemic lupus erythematosus. Arthritis Rheum 2006; 54: 2184-2197.

30 Wagner CL, Hanumanthu VS, Talbot CC, et al. Short telomere syndromes cause a primary T cell immunodeficiency. J Clin Invest 2018; 128: 5222-5234.

31 Oliveira NM, Rios EC, de Lima TM, et al. Sepsis induces telomere shortening: a potential mechanism responsible for delayed pathophysiological events in sepsis survivors? Mol Med 2017; 22: 886-891.

32 Putman RK, Hunninghake GM, Dieffenbach PB, et al. Interstitial lung abnormalities are associated with acute respiratory distress syndrome. Am J Respir Crit Care Med 2017; 195: 138-141.

33 Aubert G, Hills M, Lansdorp PM. Telomere length measurement-caveats and a critical assessment of the available technologies and tools. Mutat Res 2012; 730: 59-67.

34 Barrow PA, Desmidt M, Ducatelle R, et al. World Health Organisation-supervised interlaboratory comparison of ELISAs for the serological detection of Salmonella enterica serotype Enteritidis in chickens. Epidemiol Infect 1996; 117: 69-77.

35 Rufer N, Dragowska W, Thornbury G, et al. Telomere length dynamics in human lymphocyte subpopulations measured by flow cytometry. Nat Biotechnol 1998; 16: 743-747.

36 Seymour CW, Liu VX, Iwashyna TJ, et al. Assessment of clinical criteria for sepsis: for the Third International Consensus Definitions for Sepsis and Septic Shock (Sepsis-3). JAMA 2016; 315: 762-774. 